\title{
Mesures hydrologiques par radar
}

\section{The use of radar for hydrological measurements}

\author{
H. Sauvageot \\ Laboratoire d'Aérologie \\ Université Paul Sabatier, Toulouse
}

\section{Introduction}

La mesure des précipitations est une préoccupation majeure dans de nombreux domaines de recherches ou d'application tels que l'agriculture, l'hydrologie, la météorologie, la climatologie et les télécommunications. Toutefois, les délais dont on dispose pour réaliser et exploiter la mesure ainsi que la précision et la résolution temporelle et spatiale désirées ou l'étendue de la zone à observer diffèrent sensiblement suivant les cas. Pour les télécommunications par exemple, les besoirs portent surtout sur la connaissance des statistiques climatologiques relatives à l'occurence des précipitations en fonction de leur intensité, de leur étendue et de leur durée en vue d'évaluer les perturbations probables des liaisons hertziennes. Aucun problème d'exploitation en temps réel ne se pose. Par contre, pour la prévision opérationnelle des crues des rivières ou plus généralement des pointes de débit des réseaux d'écoulement des eaux de ruissellement, la meilleure continuité possible des observations est souhaitée; en outre, une exploitation des données en temps quasi-réel est la condition de l'efficacité.

Les problèmes pratiques de mesure en un point unique peuvent être résolus à l'aide d'un pluviomètre à intensité doté d'une télémesure. Pourtant, dans la plupart des cas pratiques, c'est une surveillance à la fois dans l'espace et le temps qui est nécessaire - la solution technique la plus évidente consiste à développer des réseaux de mesure pluviométrique au sol. Cependant, de nombreuses raisons pratiques telles que le coût, l'accessibilité (dans les zones inhospitalières) et la sutveillance (dans les zones à faible peuplement) limitent la densité de tels réseaux. La télédétection à l'aide de radars météorologiques offre une solution élégante puisque, les signaux météorologiques étant interprétables en terme d'intensité de précipitation, elle per- met une surveillance hydrologique sur de grandes étendues. Les meilleurs résultats sont obtenus en associant le radar et les mesures au sol.

Il s'agit là d'une application remarquable de la technique radar à l'observation météorologique. Sa mise au point a fait l'objet de nombreux travaux dont l'origine remonte aux tous premiers temps du radar. Diverses méthodes ont été proposées pour réaliser la mesure; elles sont fondées principalement sur les propriétés de la réflectivité radar des précipitations et sur celles de l'atténuation subie par le rayonnement au cours de la propagation dans le milieu diffusant. Chacune de ces deux quantités peut être reliée approximativement par des formules simples à l'intensité de la précipitation $(R)$; la connaissance de cette dernière en fonction du temps permet d'accéder à la quantité (ou hauteur) de précipitation atteignant une surface au cours de l'intervalle de temps pendant lequel la mesure a été faite. En réalité, la plupart des études et essais réalisés dans ce domaine font appel à la seule mesure du facteur de réflectivité $\operatorname{radar} Z$, à l'aide de longueurs d'onde peu atténuées, les mesures d'atténuation précises étant difficiles à réaliser et peu compatibles avec les conditions d'une mise en cuvre opérationnelle. Le présent article traitera essentiellement des techniques de mesure par rétrodiffusion, les techniques faisant appel à l'atténuation font l'objet d'un appendice en fin de texte.

Dans ce qui suit, on trouvera d'abord un exposé du principe des mesures à partir de notions touchant aux propriétés des précipitations et de la diffusion des ondes électromagnétiques du radar. On introduira les quantités permettant de caractériser les phénomènes. On analysera ensuite les facteurs intervenant sur la qualité des données du radar, puis ayant considéré le cas des mesures soit par radar soit par réseau pluviométrique au sol, on discutera des configurations associant ces deux types de moyens sous l'angle de l'optimisation et de la précision des résultats en fonction des caractéristiques des réseaux. 


\section{Principe des mesures hydrologiques par radar}

\subsection{Utilité et avantages de la méthode}

La méthode consiste à balayer la zone utile, à intervalles réguliers, avec le faisceau du radar dans le but de mesurer la réflectivité des précipitations puis, à l'aide des relations analytiques convenables à convertir les réflectivités en intensité de précipitation. L'intégration des intensités au cours du temps permet d'obtenir la distribution spatio-temporelle des quantités de précipitations. L'information recherchée concernant généralement la précipitation au niveau du sol, la mesure radar doit être faite aussi près que possible de la surface (balayage suivant les azimuts, à site faible). Les réflectivités sont mesurées suivant un réseau de points correspondant à des variations discrètes de distance et d'angle.

Comparée à un réseau de pluviomètres conventionnels, la mesure des précipitations par radar offre d'importants avantages parmi lesquels :

- la continuité spatiale des observations (la maille des réseaux de pluviomètres est généralement supérieure à l'échelle des cellules convectives développées donnant les précipitations intenses, lesquelles peuvent ainsi "échapper" au réseau ou l'induire en erreur par des maximums non représentatifs de l'intensité dans la maille);

- un meilleur accès à l'observation de la variabilité des précipitations

- la possibilité d'une surveillance en temps réel sur une grande étendue à partir d'un seul point de mesure; - la facilité d'acquisition, de stockage et de traitement informatique des données.

L'inconvénient majeur tient évidemment à la précision des mesures qui est notablement inférieure à la précision des mesures "in situ", comme c'est le cas pour la plupart des observations par télédétection. Il se posera donc un problème d'étalonnage.

\subsection{Caractéristiques physiques des précipitations}

Pour les mesures hydrologiques, il est commode de caractériser les précipitations par leur intensité (ou taux) $R$ défini comme étant le flux d'eau traversant une section horizontale de surface unité pendant un certain intervalle de temps. On l'exprime par une hauteur (celle du volume d'eau équivalent de section unité) divisée par un temps. $R$ est donné par l'expression :

$$
R=\frac{\pi}{6} \int_{D_{\min }}^{D_{\max }} N(D) D^{3}[v(D)-w] d D
$$

où :

- $D$ est le diamètre des hydrométéores,

- $N(D) d D$ la concentration numérique dans le volume unité,

- $v(D)$, la vitesse limite de chute des hydrométéores dont le diamètre est compris entre :

$$
D-\frac{1}{2} d D \quad \text { et } \quad D+\frac{1}{2} d D,
$$

- w la composante verticale de la vitesse de l'air comp- tée positivement vers le haut (pour les mesures au sol $w=0$ ) ;

$R$ est expriné usuellement en $\mathrm{mm} \mathrm{h}^{-1}$ d'eau liquide.

Cette relation est générale et s'applique à tout type d'hydrométéores sphériques.

$R$ peut être mesuré ou calculé à l'aide de l'expression (1). Dans ce dernier cas, on doit connaitre la répartition granulométrique de la précipitation en fonction de la taille des hydrométéores ainsi que leurs vitesses de chute. D'autres paramètres, tels que le contenu en eau $M$ ou le diamètre volumique médian $D_{0}$ sont parfois utilisés ; cependant, leur intérêt pratique est faible.

\section{Répartition granulométrique des précipitations}

La microstructure des précipitations naturelles a fait l'objet de nombreux travaux. A partir de mesures au sol, Marshall et Palmer (1948) ont établi une relation fonctionnelle de forme exponentielle entre $N(D)$ et $D$.

avec $\Lambda=a R^{b}$

$$
N(D)=N_{0} e^{-\Lambda D}
$$

où $N_{0}, a$ et $b$ sont des coefficients caractéristiques de la distribution et $R$ l'intensité de précipitation en $\mathrm{mm} h^{-1}$.

Cette relation, quant à sa forme, est acceptée et d'un usage général. Son intérêt vient de ce qu'elle n'est fonction que de la quantité $R$ qui est mesurée le plus facilement et le plus fréquemment.

Pour la pluie, la distribution de Marshall-Palmer est caractérisée par les valeurs particulières des coefficients :

$$
N_{0}=0,08 \text { et } \Lambda=41 R^{-0,21}
$$

$N(D)$ et $N_{0}$ sont en $\mathrm{cm}^{-4}$ et $\Lambda$ en $\mathrm{cm}^{-1}$

Des coefficients un peu différents ont été obtenus par d'autres auteurs. Par exemple, à partir d'observations de spectres par radar, en visée verticale, dans des précipitations à caractère stratiforme, Sekhon et Srivastava (1971) écrivent :

$$
N_{0}=0,07 R^{0,37} \text { et } \Lambda=38 R^{-0,14}
$$

avec les mêmes unités que (3).

La figure 1 donne l'allure des distributions (3) et (4). La validité de ces relations est confirmée par l'expérience, avec cependant quelques limitations. D'une part, aux petites dimensions, la distribution n'est plus exponentielle et, en deça d'un diamètre d'environ $1 \mathrm{~mm}$, des coefficients modifiés doivent être utilisés (Wexler et Atlas, 1963); cette déviation est sensible aux faibles intensités. D'autre part, les grosses gouttes ont tendance à se scinder et ne dépassent jamais un diamètre d'environ $6 \mathrm{~mm}$. Donc, d'une manière générale, des précautions sont nécessaires aux fortes intensités de préciptation. De plus, ces relations correspondent à des moyennes statistiques - en réalité, les précipitations présentent, localement et sur de courtes durées, des déviations importantes par rapport aux répartitions granulométriques indiquées, dues notamment à l'influence du cisaillement vertical de vent.

Pour les précipitations de neige, les valeurs moyennes suivantes ont été suggérées (Sekhon et Srivastava, 1970):

$$
N_{0}=0,025 R^{-0,94} \text { et } \Lambda=2,29 R^{-0,45}
$$

avec les mêmes unités que (3). 


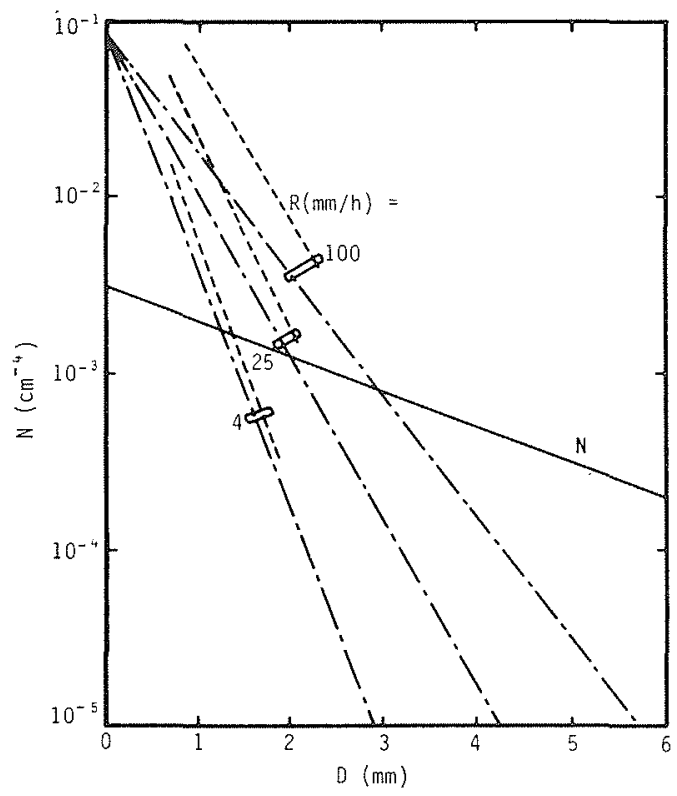

Figure 1 - Distribution de gouttes de pluie (concentration numérique en fonction du diamètre) proposées par Marshall et Palmer (trait mixte) et Sekhon et Srivastava (trait tireté) pour diverses valeurs de l'intensité de précipitation $R$. La droite $N$ représente, pour une précipitation de neige de $10 \mathrm{~mm}^{-1}$, la distribution des diamètres de particules fondues équivalentes donnée par l'expression 5.

Pour la grêle, par suite de la grande variabilité naturelle des propriétés granulométriques, il est difficile d'établir une expression de valeur universelle.

\subsection{Le capteur radar}

Le radar conventionnel émet dans l'atmosphère, à intervalles de temps égaux, des impulsions d'énergie électromagnétique puissantes très brèves et de fréquence élevée. L'énergie est concentrée en un faisceau de faible ouverture par une antenne directive. Les cibles de toutes natures présentes dans le faisceau, interceptent une partie de l'énergie incidente qu'elles absorbent et rayonnent dans diverses directions. La fraction renvoyée vers le radar est le signal utile. Si l'on admet en première approximation que, sur des distances n'excédant pas quelques centaines de kilomètres, les ondes électromagnétiques utilisées se propagent en ligne droite et à vitesse constante, l'orientation de l'antenne et le temps écoulé entre l'émission de l'impulsion et la réception du signal permettent de localiser la région diffusante en direction et en distance.

Dans les observations météorologiques, la cible est une région diffusante telle qu'une précipitation dont les dimensions sont importantes par rapport à celles du faisceau. L'observation, à toute les distances dans la direction de visée, combinée aux mouvements de l'antenne permet d'en faire une exploration en volume.

\section{Propriétés statistiques du signal}

Les zones cibles considérées sont remplies par un grand nombre de diffuseurs indépendants (gouttelettes d'eau, cristaux de glace, etc.). Pour une impulsion de longueur finie $h$ (avec $h=C$ r où $C$ est la vitesse de pro- pagation électromagnétique et $\tau$ la largeur temporelle de l'impulsion), le signal reçu pour une distance quelconque $r$ est la somme des signaux renvoyés vers le radar (rétrodiffusés) par chaque diffuseur présent dans le volume d'impulsion ou d'échantillonnage $V$. Ce volume, qui définit la résolution spatiale de la mesure, est habituellement de forme à peu près cylindrique (faisceau conique de faible ouverture). Sa résolution latérale à la distance $r$ est $r \theta$ où $\theta$ est l'ouverture du faisceau et sa résolution radiale à toute distance est $\frac{C \tau}{2}$ (le front de l'onde rétrodiffusée par les particules situées à la distance $r+C \tau / 2$ parviennent au radar en même temps que l'arrière de l'onde rétrodiffusée par les particules situées à la distance $r$ et, par raison de continuité, en même temps aussi que les contributions de toutes les particules situées entre ces deux distances). Les valeurs courantes de $\theta$ et de $\tau$ sont autour de $1^{\circ}$ et $1 \mu$ sec.

Le signal reçu par le radar à l'instant $t$ et pour la distance $r$ est la somme des ondes renvoyées par chaque diffuseur situé dans le volume d'échantillonnage (usuellement plus de $10^{5}$ diffuseurs indépendants). Ces ondes ont des amplitudes différentes (dépendant de la taille, de la forme, de l'état physique, de la température et des propriétés diélectriques du diffuseur) et des phases variables en fonction de la position des diffuseurs. En effet, les diffuseurs se déplacent les uns par rapport aux autres et par rapport au radar. Les mouvements sont dus au vent moyen, à la turbulence de petite échelle, au cisaillement du vent, à la chute des diffuseurs et aux différences entre les vitesses de chute. Le nombre de diffuseurs intervenant simultanément étant très grand, on montre que la résultante a une phase dont la statistique est uniforme entre 0 et $2 \pi$ et que la densité de probabilité de lamplitude est une distribution de Rayleigh. Donc, dans un radar météorologique observant une distribution de cibles indépendantes telle qu'une précipitation, le signal reçu se présente comme une fonction aléatoire du temps résultant de l'échantillonnage d'un processus gaussien. Cette fonction ne peut être décrite que statistiquement à partir de sa densité spectrale de puissance et de ses moments. Les principaux paramètres utiles sont la puissance moyenne du signal reçu $P_{r}$, la vitesse moyenne de la cible $V$, la variance du spectre $\sigma^{2}$ et dans certaines applications le spectre lui-même

Les mesures hydrologiques par radar dérivent de la mesure de la puissance du signal reçu. Cette quantité, de même que la variance du spectre peuvent être déterminées à l'aide d'un radar météorologique conventionnel, c'est-à-dire sans détermination des relations de phase entre l'onde émise et le signal reçu. Par contre, la vitesse moyenne et le spectre de vitesse de la cible ne sont accessibles qu'au radar cohérent par la mesure de l'effet Doppler affectant le signal. Il n'est donc pas nécessaire de disposer d'un radar cohérent (Doppler) pour procéder à des mesures hydrologiques. Toutefois, les radars Doppler permettent de déterminer la distribution des tailles d'hydrométéores précipitants au-dessus du radar à partir de la mesure des spectres de vitesse Doppler par visée verticale. Ils donnent aussi accès à de nombreuses informations sur la structure du champ dynamique local. Dans ce qui suit, nous 
n'envisagerons que le cas de mesures à l'aide d'un radar conventionnel.

\section{L'équation du radar}

Le signal reçu par le radar est lié aux propriétés diffusantes de la précipitation observée par une équation de bilan d'énergie tenant compte des caractéristiques techniques de l'équipement; c'est l'équation du radar (voir par exemple Sauvageot, 1982). Pour un volume diffusant situé à la distance $r$ du radar, la puissance moyenne du signal reçu $\bar{P}_{r}$ s'écrit :

$$
\bar{P}_{r}=C_{1} L^{2} \eta / r^{2}
$$

où :

- $C_{1}$ est une constante dépendant uniquement des caractéristiques techniques du radar utilisé (à savoir puissance émise, longueur d'onde, gain d'antenne et forme du faisceau, largeur et forme de l'impulsion émise).

- $L^{2}$ exprime la perte d'énergie (ou atténuation) par absorption et diffusion pour le trajet aller-retour entre le radar et la cible. On a $L^{2}=10^{-0,2} \int_{0}^{r} a d r$ où $a$ est le coefficient d'atténuation exprimé en décibels $(d B)$ par unité de longueur. L'atténuation dépend de la longueur d'onde.

- $\eta$ est la réflectivité radar qui ne dépend que des pro. priétés diffusantes de la cible; elle est égale à la valeur moyenne, rapportée à un volume unité, de la section efficace de rétrodiffusion de la cible occupant le faisceau du radar à la distance $r$.

Dans la plupart des cas pratiques, les mesures hydrologiques par radar sont faites avec des radars de longueur d'onde voisine de $10 \mathrm{~cm}$ pour lesquels l'atténuation $L^{2}$ est négligeable. Donc il n'en sera pas tenu compte dans ce qui suit. Cependant, dans le cas où ce terme n'est pas négligeable, il peut être évalué approximativement en intégrant l'équation (6) le long de la trajectoire des ondes dans le milieu atténuant, le coefficient $a$ étant alors exprimé en fonction de $\eta$ ou d'une quantité dont la relation avec $\eta$ est connue.

\subsection{La rétrodiffusion par les hydrométéores}

Lorsque le rapport de la taille des diffuseurs à la longueur d'onde utilisée est très inférieur à 1 , la diffusion des ondes électromagnétiques par les particules se situe dans le domaine de diffusion de Rayleigh. C'est le cas des hydrométéores (excepté la grêle) aux longueurs d'ondes utilisées pour l'observation des précipitations par radar (entre 3 et $10 \mathrm{~cm}$ ). Dans ces conditions, la section efficace de rétrodiffusion radar $(\sigma)$ d'un diffuseur sphérique et homogène est donnée par les formules dites de l'approximation de Rayleigh, à savoir :

$$
\sigma=\frac{\pi^{5}}{\lambda^{4}}|K|^{2} D^{6}
$$

où :

- $D$ est le diamètre du diffuseur,

- $\lambda$ la longueur d'onde,

- et $|K|^{2}$ est le facteur diélectrique du matériau constituant la sphère ; sa valeur approximativement est de 0,93 pour l'eau et de 0,18 pour la glace (pour une densité de $0,92 \mathrm{~g} \mathrm{~cm}^{-3}$ ).

La réflectivité radar d'une population d'hydrométéores sphériques dont les propriétés physiques et la répartition sont homogènes au sein du volume de résolution est donnée par la relation:

$$
\eta=\sum_{i} \sigma_{i}=\frac{\pi^{5}}{\lambda^{4}}|K|^{2} \sum_{i} D_{i}^{6}
$$

la sommation portant sur le volume unité.

\section{Le facteur de réflectivité radar}

La réflectivité radar (éq. 8) est donc proportionnelle à la somme de la puissance 6 des diamètres des hydrométéores. Le terme $\sum_{i} D_{i}^{6}$ est appelé le facteur de réflectivité radar et représenté par la lettre $Z$. On peut écrire :

$$
Z=\int_{D_{\min }}^{D_{\max }} N(D) D^{6} d D
$$

Tout comme $\eta$, le facteur $Z$ est une propriété moyenne de la population de diffuseurs répartie dans le volume d'impulsion. $Z$ doit normalement être exprimé en $\mathrm{m}^{6} \mathrm{~m}^{-3}$; cependant, la taille des éléments de précipitations étant de l'ordre du millimètre, il est donné usuellement en $\mathrm{mm}^{6} \mathrm{~m}^{-3}$ (avec un facteur de conversion de $10^{18} \mathrm{~mm}^{6} / \mathrm{m}^{6}$ ). A cette unité, on associe une échelle logarithmique définie par comparaison avec un niveau de référence de $1 \mathrm{~mm}^{6} \mathrm{~m}^{-3}$. Le facteur de réflectivité radar est ainsi exprimé en $d B Z$ :

$$
Z(d B Z)=10\left[\log Z\left(\mathrm{~mm}^{6} \mathrm{~m}^{-3}\right)\right]
$$

Lorsque le volume diffusant observé ne remplit pas les conditions d'approximation de Rayleigh, ou qu'il existe un doute à ce sujet, il est commode de le caractériser par le facteur de réflectivité équivalent $Z_{e}$ qui est égal à celui d'une population de particules liquides et sphériques satisfaisant à l'approximation et produisant un signal de même puissance.

Le facteur de réflectivité radar $Z$ offre l'avantage de n'être pas influencé par la longueur d'onde; il se prête donc mieux aux comparaisons entre des mesures obtenues avec des équipements différents.

L'équation (6) peut donc s'écrire :

$$
\bar{P}_{r}=C_{2} L^{2}|K|^{2} \frac{Z}{r^{2}}
$$

où $C_{2}$ est une constante dépendant du radar utilisé et des unités.

Rappelons que l'équation $(10)$ s'applique à une cible distribuée lorsque sont satisfaites les hypothèses suivantes :

1) la cible occupe tout le volume de résolution de l'impulsion ;

2) elle est composée de particules sphériques ou assimilables à des sphères (pour la pluie, des déformations apparaissent pour les diamètres supérieurs à environ $1 \mathrm{~mm}$ ) ;

3) la taille des particules est faible vis-à-vis de la longueur d'onde et satisfait les conditions de l'approximation de Rayleigh ; 
4) les propriétés diélectriques $\left(|K|^{2}\right)$ et granulométriques (Z) sont homogènes dans le volume considéré ;

5) l'antenne est gaussienne;

6) sa polarisation est linéaire ;

7) les effets de diffusion multiple sont négligeables.

Lorsque la condition 1 n'est pas respectée, l'équation (10) n'est utilisable que si l'on sait déterminer un coefficient correctif d'occupation du faisceau exprimant la fraction du volume d'impulsion occupée par la cible, pondérée par la valeur relative du gain de l'antenne dans l'angle solide correspondant.

Lorsque les conditions 2,3 et 4 ne sont pas satisfaites, $Z$ est remplacé par le facteur de réflectivité équivalent $Z_{e}$ avec $|K|^{2}=0,93$.

\section{Relations $Z-R$ et $Z-M$ pour les précipitations.}

Les précipitations naturelles satisfont aux conditions de l'approximation de Rayleigh pour les longueurs d'ondes centimétriques et décimétriques (excepté la grêle) et la forme approximative de leur distribution dimensionnelle est donnée par une expression analytique dont le paramètre $R$ est aussi la principale propriété utile. On est donc fondé à rechercher des relations simples entre $Z$ et $R$. De nombreux travaux ont été consacrés à l'étude de telles relations. Leur forme générale est :

$$
Z=a R^{b}
$$

où $a$ et $b$ sont des constantes qui "spécialisent" la relation.

La valeur des constantes est déterminée expérimentalement de plusieurs façons : à partir de mesures des distributions granulométriques de particules par spectrogranulomètre ou par radar Doppler en visée verticale. Ces distributions permettent le calcul de $Z$ et $R$, puis, l'ajustement d'une courbe de régression aux valeurs mesurées donne $a$ et $b$. Lorsque la mesure est faite par radar, $Z$ est aussi déterminé directement. La mesure simultanée en un même lieu de $Z$ par radar conventionnel et de $R$ à l'aide d'un ou plusieurs pluviomètres à faible temps de réponse, permet également d'obtenir les couples de valeurs; toutefois, on ne peut, dans ce cas, connaître la distribution granulométrique de la précipitation et s'assurer de sa régularité. Ces diverses techniques sont souvent utilisées simultanément et donnent lieu à comparaison.

Bien que d'un point de vue physique il n'y ait pas de différence fondamentale entre les types de précipitations, qui toutes résultent d'un processus de croissance par coalescence ou accrétion associé à un mouvement vertical ascendant, les conditions particulières aux diverses espèces de nuages, de même que les conditions climatologiques créent des particularités que reflètent les valeurs expérimentales correspondantes des constantes $a$ et $b$ (la qualité des mesures intervient aussi, pour partie, sur la dispersion des valeurs observées).

Un grand nombre de relations expérimentales (plus de cent) ont été publiées (voir parmi d'autres, Marshall et Palmer, 1948; Fujiwara, 1965; Dumoulin et Cogombles, 1966; Cataneo et Stout, 1968; Stout et Mueller, 1968-Joss et al, 1970; Sekhon et Srivastava, 1970; Wilk et Kessler, 1970). Parmi les relations expé- rimentales les plus significatives obtenues à partir de mesures de spectres granulométriques, on peut retenir :

- pour les pluies convectives (Jones, 1956):

$$
Z=486 R^{1,37}
$$

- pour les pluies à caractères stratiforme (Marshall et Palmer, 1948) :

$$
Z=200 R^{1,6}
$$

- pour la neige (Sekhon et Srivastava, 1970) :

$$
Z=1780 R^{2,21}
$$

avec $Z$ en $\mathrm{mm}^{6} \mathrm{~m}^{-3}$ et $R$ en $\mathrm{mm} \mathrm{h}^{-1}$.

Ces formules ne s'appliquent que lorsque les conditions d'approximation de Rayleigh sont satisfaisantes. Pour un même type de nuage et un même lieu, des écarts importants sont observés et l'estimation de l'une des deux quantités à partir de la mesure de l'autre, à l'aide de ces expressions n'est significative que pour des valeurs moyennes résultant d'une intégration suffisante dans l'espace et le temps. Les écarts les plus importants sont trouvés pour les intensités faibles et très fortes. Pour les intensités moyennes, ces diverses relations donnent des résultats acceptables (voir $\S 3$ ).

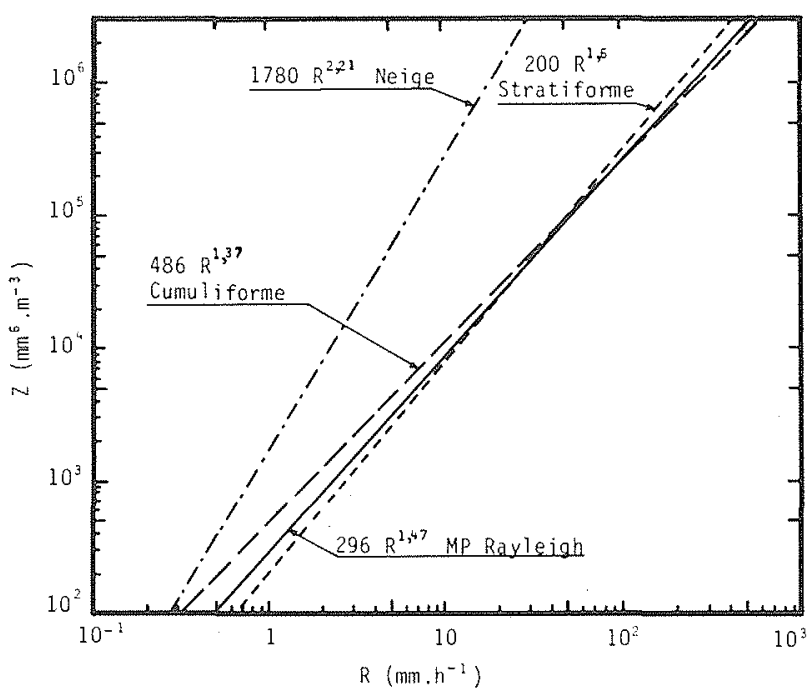

Figure 2 - Relation entre le facteur de réflectivité radar $Z$ et l'intensité de précipitation $\dot{R}$ correspondant à divers types de distribution de taille de gouttes de précipitations.

Sur la figure 2 sont indiquées les trois relations expérimentales relatives à la neige et aux pluies d'origine stratiforme et cumuliforme (éq.. 12 à 14), ainsi que la relation correspondant à une distribution granulométrique de Marshall-Palmer diffusant selon la loi de Rayleigh $\left(Z=296 R^{1,47}\right)$.

On remarque que la courbe relative à la neige est située au-dessus des autres; cela traduit le fait que, par suite de la faible vitesse limite de chute de la neige, à une intensité de précipitation donnée correspond dans l'air un contenu en eau plus grand pour la neige que pour la pluie.

Des formules analogues aux relations entre $Z$ et $R$ peuvent être établies entre $Z$ et $M$ dans les précipitations. 


\section{Mesures hydrologique par rétrođiffusion}

\subsection{Utilisation de la mesure radar pour déterminer $R$}

On a vu que les variations de $Z$ et $R$ dans les précipitations sont liées approximativement par des relations de forme $Z=a R^{b}$. Les coefficients $a$ et $b$ de ces relations dépendent de la nature et des caractéristiques granulométriques des précipitations observées. $Z$ est proportionnel à $D^{6}$ tandis que $R$ est proportionnel au produit du volume des particules en précipitation (proportionnel à $D^{3}$ ) par leur vitesse de chute (proportionnel à une puissance de $D$ comprise entre 0 et 2). L'influence de ce dernier terme conduit à des valeurs de $b$ comprises approximativement entre 1,2 et 2,2 (éq. 12 à 14).

Lorsque l'atténuation est négligeable, l'équation (10) peut être écrite :

$$
\bar{P}_{r}=C_{3} \frac{a R^{b}}{r^{2}}
$$

où $C_{3}$ est une constante dépendant du radar, des unités et du facteur diélectrique des particules en précipitation. La valeur de $R$ est donc :

$$
R=\left(\frac{r^{2}}{a C_{3}} \bar{P}_{r}\right)^{b-1}
$$

La "mesure" peut être faite à l'aide des seules données radar; cependant, les meilleurs résultats sont obtenus en déterminant expérimentalement les "facteurs d'étalonnage" de la relation (15) (i.e. $C_{3}$, $a$ et $b$ ) (Harrold et al., 1974). Pour ce faire, on doit :

- soit mesurer la distribution granulométrique de la précipitation dont on déduit des couples $Z-R$, - soit comparer $\Sigma R$ mesuré pendant un intervalle de temps donné par un pluviomètre sur le terrain avec les valeurs correspondantes de $\Sigma Z$ ou $\Sigma \bar{P}_{r}$ mesurées pendant le même temps par le radar aussi près que possible du pluviomètre.

\subsection{Qualité des mesures du radar}

La qualité des mesures est tributaire d'un éventuel réseau d'étalonnage mais aussi de nombreux autres facteurs météorologiques ou instrumentaux. Parmi ces derniers, l'ouverture du faisceau est primordiale en ce qu'elle détermine la résolution spatiale du radar; en effet, les dimensions du volume d'impulsion fixent la taille minimale des variations de petite échelle accessibles au radar donc son aptitude à mesurer la valeur exacte des extremums locaux; plus généralement, la résolution intervient dans les problèmes liés à l'homogénéité de la cible et mentionnés à propos de l'équation (10) comme condition d'application quantitative de l'équation du radar météorologique. En effet, un faisceau dont l'ouverture a la valeur courante de $1^{\circ}$ correspond à un volume de résolution dont la dimension latérale est de $2,6 \mathrm{~km}$ à $150 \mathrm{~km}$. C'est là une dimension maximale admissible dans les cas pratiques si l'on considère que la condition de régularité de la précipitation concerne l'homogénéité horizontale de la distribution granulométrique (en tant que résultat d'un même pro- cessus physique de formation), mais aussi l'homogénéité verticale des propriétés du milieu diffusant. Par exemple, dans le cas d'une pluie formée à partir d'une précipitation de neige (ce qui est le cas général dans les nuages à caractère stratiforme), la présence de la couche de fusion (bande brillante) dans le volume de précipitation observé introduit de sérieuses erreurs. D'autres aspects purement météorologiques interviennent, parmi lesquels les modifications de la précipitation pendant sa chute vers le sol, au sein de la couche dans laquelle est faite la mesure ; ainsi en est-il avec l'évaporation partielle dans de l'air sec, entre le nuage et le sol ou, au contraire, de la croissance dans de l'air nuageux éventuellement en présence de vitesses verticales ascendantes non négligeables (lesquelles s'annulent à très bas niveau).

Les autres problèmes critiques des mesures hydrologiques par radar sont ceux des échos parasites (échos de sol en particulier) et surtout des masques occultant éventuellement une partie du faisceau; ils sont liés à la configuration du terrain, à l'implantation du radar et à la forme du faisceau. La figure 3 représente la précision des mesures d'un radar de "performances moyennes" étalonné par un pluviomètre en fonction de la distance. On voit que la précision demeure acceptable jusqu'à 100 à $150 \mathrm{~km}$, puis se dégrade rapidement au-delà,

En résumé, les principales sources d'erreur pouvant intervenir dans les mesures hydrologiques par radar sont :

- les défauts d'homogénéité de la cible :

- précipitations de types différents (par exemple solide et liquide),

- anomalies de la distribution granulométrique : distribution à plusieurs modes ou présence de particules situées hors du domaine de Rayleigh (grêle),

- faisceau rempli de façon non uniforme,

- présence de la couche de fusion,

- présence de cibles parasites.

- modification de la précipitation entre le sol et la région où est faite la mesure radar :
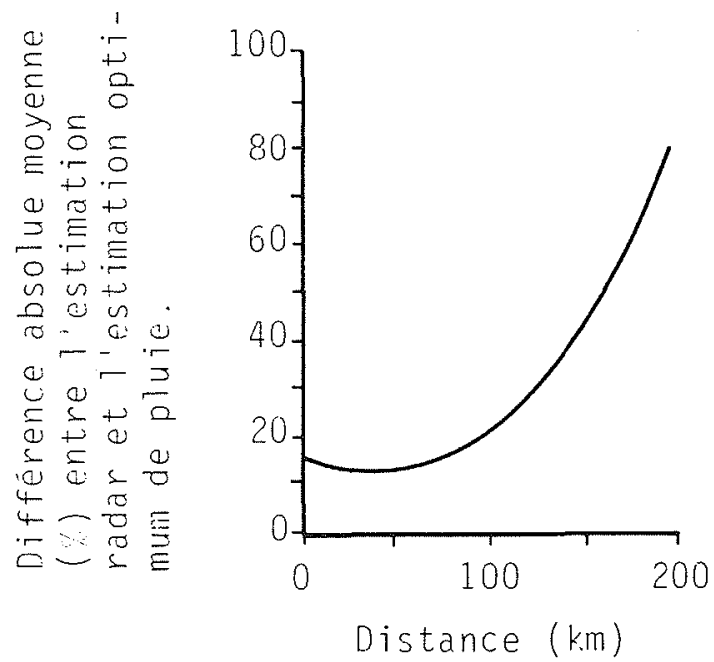

Figure 3. - Précision des mesures de précipitation d'un radar étalonné par un pluviomètre, en fonction de la distance (d'après Collier, 1975). 
- évaporation ou croissance,

- mouvements verticaux de l'air,

- défaut d'étalonnage :

- erreur sur la mesure de pluie du pluviomètre (par exemple effet du vent),

- erreur sur la mesure radar (par exemple présence d'eau sur le radôme ou sur le réflecteur parabolique).

- problèmes de propagation:

- atténuations (précipitations, nuages, gaz),

- propagation anormale,

- masques.

- périodicité des mesures inadaptées au type de précipitation observé.

- défaut dans le traitement des données, en particulier procédé de mesure de l'intensité moyenne du signal radar et de calcul de la moyenne dans le temps et l'espace.

Parmi ces causes d'erreur, certaines peuvent être corrigées ou évitées grâce à des précautions expérimentales convenables. Les erreurs dues à l'atténuation pour une mesure de précipitation par rétrodiffusion semblent par contre très difficiles à calculer et entraînent des incertitudes prohibitives sur les mesures. On est donc conduit à utiliser pour ces mesures des ondes relativement longues. Il est admis que $10 \mathrm{~cm}$ de longueur d'onde est un compromis favorable pour les mesures de précipitation par rétrodiffusion. La distance d'observation intervient aussi sur la précision-cette dernière demeure à peu près constante jusqu'à une distance de quelques dizaines de kilomètres (dépendant de l'ouverture du faisceau, de la longueur d'onde, etc.) puis se détériore rapidement ensuite.

\subsection{Mesure des précipitations à l'aide des seules données du radar.}

Un problème se pose avec cette méthode, concernant le choix des constantes de la formule de conversion $Z-R$ que l'on doit utiliser et dont dépend la précision des estimations de précipitation. En effet, les propriétés des précipitations, c'est-à-dire les facteurs $a$ et $b$ de la relation $Z-R$, varient dans l'espace et le temps (voir par exemple Drufuca, 1977). Elles dépendent, comme on l'a vu, de diverses conditions telles que le type de nuage, les effets locaux particuliers (orographiques, côtiers, etc.) soit de façon générale des processus microphysiques et cinématiques affectant la distribution granulométrique et la vitesse de chute des éléments de précipitation (par exemple la déposition et l'évaporation, l'accrétion, la coalescence, l'aggrégation et le fractionnement, le triage par le cisaillement de vent, les courants verticaux), Les causes des variations observées des facteurs $a$ et $b$ de la relation (11) sont nombreuses et incomplètement analysées. Il apparaît globalement que le coefficient $a$ augmente et que l'exposant $b$ diminue lorsque l'intensité de la convection agissant sur le développement de la précipitation augmente (Carbone et Nelson, 1978).

Lorsque l'on ne dispose que des informations du radar, on peut s'efforcer, à partir des divers éléments d'appréciation de nature climatologique ou circonstanciels auxquels on peut accéder, de choisir "au mieux" la relation particulière susceptible de s'appliquer à la précipitation observée. Par exemple : précipitation de front chaud ou de front froid, précipitation de neige, de pluie, etc. On obtient ainsi quelques améliorations par rapport à l'emploi systématique d'une relation unique dans tous les cas, qui est la procédure la plus simple. Cependant, les possibilités offertes par cette méthode semblent limitées. En effet, les variations pouvant exister au sein d'un même type de précipitation demeurent importantes.

Pour tenter d'affiner le choix des facteurs à utiliser, on a recherché empiriquement d'éventuelles corrélations au sein d'un même type entre : d'une part, la structure des échos (considérés du point de vue de leur classification par reconnaissance de formes) et leurs propriétés physiques (telles que l'intensité moyenne ou la variance) et d'autre part, les valeurs optimales des facteurs de la formule de conversion; les liens observés sont faibles (Puhakka, 1974, parmi d'autres).

Il a aussi été proposé de se référer à la mesure de la réflectivité radar différentielle entre des ondes polarisées verticalement et horizontalement, laquelle dépend des écarts de sphéricité des gouttes de pluie et peut être reliée de façon approximative aux paramètres de la distribution granulométrique si l'on suppose que celle-ci est exponentielle (Seliga et Bringi, 1976). Par ailleurs, l'emploi simultané de deux longueurs d'ondes différentes (toutes deux non atténuées) permet d'obtenir des résultats améliorés par rapport à un dispositif à fréquence unique (Cartmill, 1963; Sulakvelidze et Dadali, 1968).

Globalement, la mesure des hauteurs de précipitation conduite dans de bonnes conditions techniques à l'aide des seules données du radar, sans étalonnage par des mesures pluviométriques simultanées au sol, permet d'obtenir des estimations situées à l'intérieur d'un facteur 2 de la vraie valeur dans environ $75 \%$ des cas (Woodley et al., 1975). Malgré cette médiocre précision, les mesures de ce type, par leur souplesse et leur simplicité de mise en oeuvre, offrent d'intéressantes possibilités d'application chaque fois qu'une mesure approximative suffit (par exemple, dans les dispositifs de surveillance hydrologique générale et pour un premier niveau d'alerte aux crues).

\subsection{Mesure des précipitations à l'aide d'un réseau de pluviomètres}

Les mesures granulométriques au sol utilisent parfois des appareils de type spectro-granulomètre qui permettent de connaître le nombre des particules en fonction de leur taille et comportent éventuellement une télémesure (voir parmi d'autres: Joss et Waldvogel, 1967; Donnadieu, 1974; Klaus, 1976 et sur les erreurs d'échantillonnage : Joss et Waldvogel, 1969 ; Gertzman et Atlas, 1977).

Les mesures pluviométriques courantes utilisent des "pluviomètres à intensité" donnant une information chaque fois qu'un certain "quantum" de précipitations a été collecté (il existe de nombreuses variantes et divers degrés de sophistication dans les techniques de mesure, d'enregistrement ou de transmission des informations). Ces appareils simples et robustes mesurent l'intensité moyenne de la précipitation pendant le temps de col- 
lecte d'un quantum (le quantum peut varier suivant les appareils entre une goutte calibrée et quelques décilitres de même que varie la surface de collection) avec une erreur dont la cause majeure est la turbulence liée à l'écoulement de l'air autour du capteur; l'erreur est une sous-estimation d'environ $10 \%$ pour un vent de $5 \mathrm{~ms}^{-1}$, $20 \%$ à $10 \mathrm{~ms}^{-1}, 20$ à $40 \%$ entre 10 et $30 \mathrm{~ms}^{-1}$.

L'erreur relative commise dans la mesure de la distribution spatio-temporelle au sol d'une précipitation par échantillonnage à l'aide d'un réseau de pluviomètres dépend de la structure de la précipitation observée et des caractéristiques du réseau utilisé ; elle varie comme l'espacement des points de mesure (la maille) du réseau et diminue lorsque l'intensité de la précipitation, l'étendue du réseau et la durée de l'événement considéré augmentent (Huff, 1971). Par exemple, une erreur moyenne inférieure à $5 \%$ est commise dans l'estimation de la quantité de précipitation due à un orage sur une étendue de $1000 \mathrm{~km}^{2}$ avec un réseau comportant un point de mesure pour $65 \mathrm{~km}^{2}$ et pour des quantités de précipitation supérieures à $1 \mathrm{~mm}$ (Huff, 1970), cependant des erreurs plus fortes ont été mentionnées dans le cas d'orage de masse d'air tropical (Woodley etal., 1975).

Les réseaux de pluviomètres utilisés pour tester les mesures radar ont en moyenne une densité de un point de mesure pour 10 à $20 \mathrm{~km}^{2}$; dans de telles conditions et excepté dans le cas de petits orages locaux ou en présence de fortes valeurs de la vitesse d'écoulement de l'air, l'erreur dans la mesure de la quantité de précipitation avec un réseau de pluviomètres est inférieure à $5 \%$.

\subsection{Mesure par radar et pluviomètres}

Un réseau très dense de pluviomètres est donc un moyen satisfaisant pour mesurer les propriétés des précipitations au sol. Mais il fait généralement défaut; l'information radar peut être considérée soit comme une donnée supplémentaire permettant d'interpoler entre les points de mesures du réseau au sol pour accroître la précision des mesures, soit comme un moyen de réduire

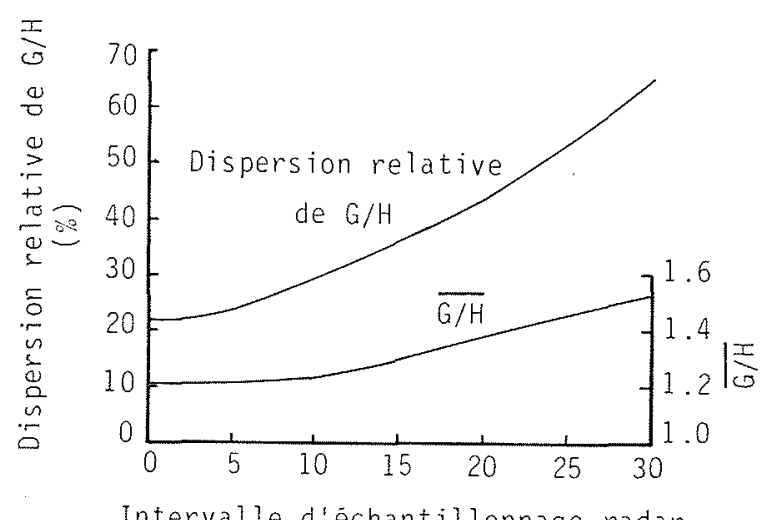

Intervalle d'échantillonnage radar (mn)

Figure 4 - La courbe du bas représente la variation de la valeur moyenne du rapport de la hauteur de pluie mesurée par un pluviomètre à la hauteur de pluie mesurée par un radar $(G / H)$ dans le cas d'une pluie d'orage, en fonction de l'intervalle d'échantillonnage du radar. La courbe du haut indique la dispersion relative des valeurs (d'après Wilson et Brandes, 1979).

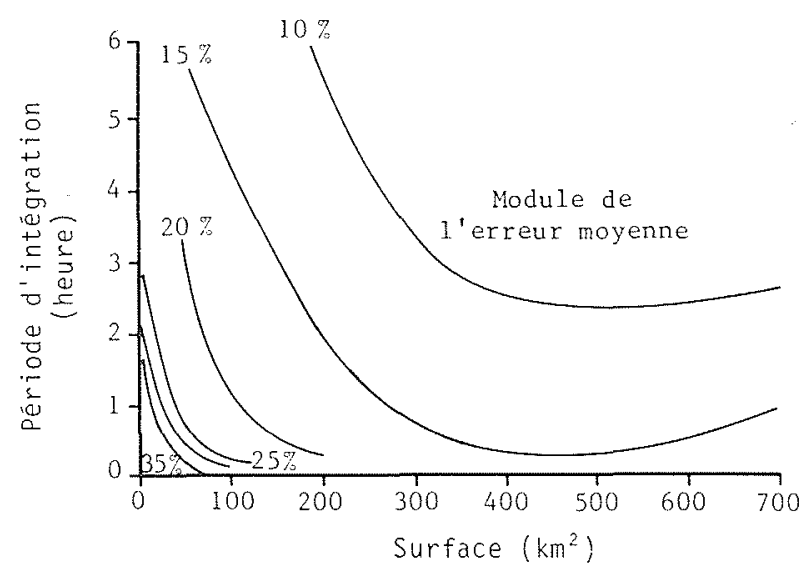

Figure 5 - Module de l'erreur moyenne (en pourcentage) entre l'estimation de quantité de pluie optimale et celle donnée par un radar étalonné à l'aide d'un seul point d'étalonnage, en fonction de la durée d'intégration et de la surface observée (d'après Collier, 1975).

la densité du réseau au sol (ou même de le supprimer par endroit), soit encore comme un moyen d'étendre les mesures au-delà du réseau au sol.

La représentativité et la validité dans le temps et l'espace d'un tel étalonnage dépendent des conditions particulières de l'observation et notamment de la structure des précipitations et de la nature du terrain, c'est-àdire de l'homogénéité des précipitations dans la zone observée. Pratiquement, pour réaliser de telles mesures dans de bonnes conditions, il est nécessaire de disposer d'un réseau de points de mesures pluviométriques d'étalonnage au sol couvrant convenablement la région étudiée et d'étalonner à une fréquence suffisante pour se prémunir contre les changements de caractéristiques des précipitations.

En effet, la validité de l'étalonnage radar diminue au fur et à mesure que le volume diffusant considéré s'éloigne du point d'étalonnage; ainsi, il est préférable de répartir les points d'étalonnage dans toute la région observée. Les caractéristiques optimales de ce réseau dépendent de la précision et de la résolution spatiotemporelle souhaitée ainsi que du type de précipitation observé et des particularités du terrain.

Les mesures tant du radar que du réseau pluviométrique sont faites par échantillonnage. De ce fait, les structures dont l'échelle est inférieure au double de la maille d'échantillonnage ne sont pas résolues. Lorsqu'elles sont échantillonnées de façon différente par le radar et le réseau, elles tendent à décorréler les mesures.

La figure 4 montre les effets de l'intervalle d'échantillonnage du radar sur le rapport $G / H$ de la hauteur de pluie mesurée par un pluviomètre à la hauteur de pluie mesurée par un radar dans le cas d'une pluie d'orage. La courbe du bas représente l'évolution de la valeur moyenne de $G / H$ en fonction de l'intervalle d'échantillonnage tandis que la courbe du haut indique la dispersion relative des valeurs du rapport (d'après Wilson et Brandes, 1979).

La figure 5 établie à partir d'observations, montre le module de l'erreur moyenne entre l'estimation de la quantité totale de pluie optimale et celle donnée par un radar étalonné à l'aide d'un seul point d'étalonnage, en fonction de la durée d'intégration de la pluie mesurée 
et de la surface observée. Le radar était réétalonné chaque heure. Cette figure montre que pour une période d'intégration donnée, l'erreur de mesure de la quantité totale de pluie décroît avec l'augmentation de la surface. Pour les petites surfaces, les fluctuations du vent réduisent la précision. Avec l'augmentation de la surface, les erreurs dues à la représentativité du pluviomètre d'étalonnage tendent à s'annuler; cependant, si la surface augmente, l'étalonnage est de moins en moins représentatif et l'erreur augmente à nouveau. La représentativité de l'étalonnage diminue aussi avec la distance par suite de l'augmentation des dimensions du faisceau. L'erreur diminue également avec la durée d'intégration; les mesures instantanées sont peu précises.

La figure 6 , déduite d'expérimentations, montre la différence moyenne entre l'estimation optimale et l'estimation donnée par un réseau de pluviomètres sans radar et pour un radar étalonné par un réseau de pluviomètres en fonction de la densité du réseau sur une étendue de $1000 \mathrm{~km}^{2}$ pour différents types de systèmes pluvieux et pour une durée de mesures de 1 heure. Il apparait clairement que pour une superficie observée de $1000 \mathrm{~km}^{2}$ et sur 1 heure, l'association radar-pluviomètre permet une précision supérieure à $20 \%$ avec un seul point d'étalonnage. L'association radar-pluviomètre est en outre très supérieure dans le cas de précipitation de type averse (convection profonde). Avec les averses typiques, un radar étalonné par 2 pluviomètres donne la même précision qu'un réseau de 50 pluviomètres sur $1000 \mathrm{~km}^{2}$.

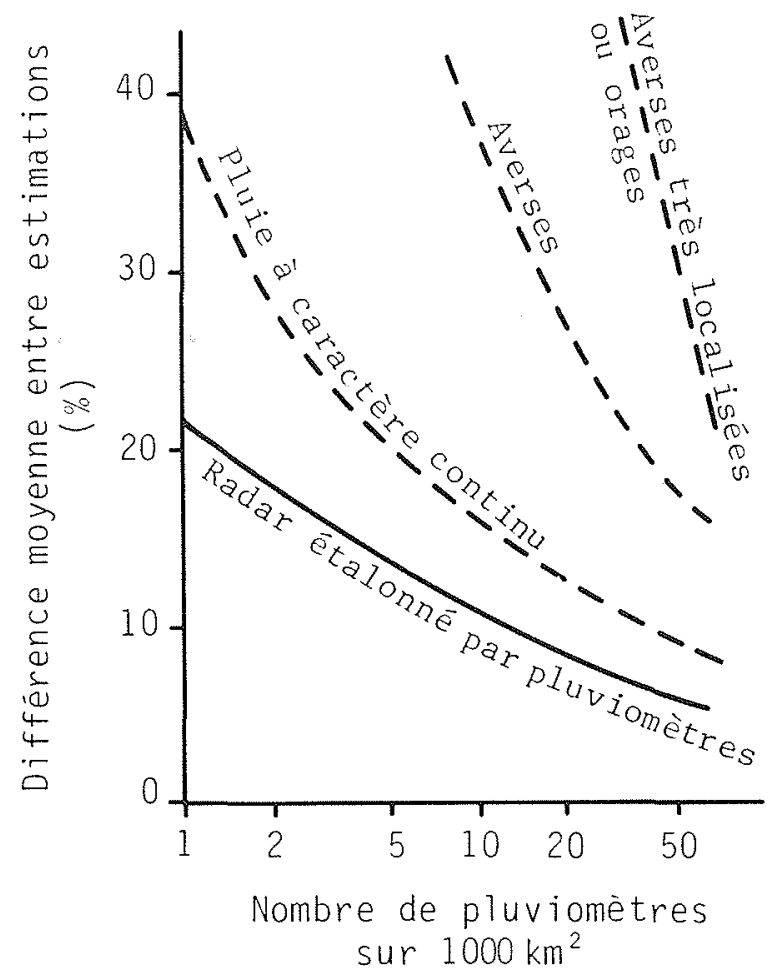

Figure 6 - Différence moyenne entre l'estimation de pluie horaire optimale et l'estimation de pluie horaire donnée par un réseau de pluviomètres sans radar (trait pointillé) et par un radar étalonné par un réseau pluviomètres (trait continu) en fonction de la densité du réseau de pluviomètres, pour une étendue de $1000 \mathrm{~km}^{2}$ et pour différents types de systèmes pluvieux (d'après Collier, 1975).

\subsection{Techniques d'ajustement des mesures de précipita- tion par radar à l'aide des données pluviométriques au sol}

La comparaison entre les valeurs moyennes des mesures de précipitation faites par radar et par un réseau pluviométrique fait apparaître des différences importantes au cours d'un même événement ou d'une précipitation à une autre; le rapport $(G / H)$ où $G$ et $H$ sont les hauteurs d'eau mesurée par le réseau pluviométrique et par le radar respectivement est fréquemment affecté d'un facteur 2 d'un orage à un autre. Il importe donc de rechercher une amélioration par un ajustement des facteurs de la conversion des données radar en intensité de précipitation. Cet ajustement peut être obtenu à partir de la confrontation des données radar et des données du réseau pluviométrique, soit en modifiant les coefficients de la relation $Z$ - $R$ utilisée, soit en conservant ces coefficients et en ajustant les estimations du radar.

\section{a) Ajustement d'une précipitation à une autre}

Dans la procédure d'étalonnage la plus simple, la différence moyenne entre les mesures radar et les mesures du réseau pluviométrique est déterminée et ure correction est appliquée uniformément aux estimations du radar. Le facteur d'ajustement multiplicatif (F) est donné par :

$$
F=\sum_{i=1}^{N} G_{i} / \sum_{i=1}^{N} H_{i}
$$

ou par :

$$
F=\frac{1}{N} \sum_{i=1}^{N} G_{i} / H_{i}
$$

Avec l'équation (17), les données sont pondérées proportionnellement à la hauteur de pluie tandis qu'avec l'équation (18) tous les couples de valeurs pluviomètreradar ont un poids égal. Dans chaque cas, les corrections concernent l'erreur associée à l'étalonnage du radar et l'erreur due à la relation $Z-R$.

\section{b) Ajustement spatial}

Les différences entre les mesures au sol et les estimations du radar ont des causes physiques qui varient dans l'espace et dans le temps. Donc, un ajustement plus précis que celui résultant de l'utilisation d'un facteur moyen peut être obtenu en se référant à un point de mesure pluviométrique suffisamment proche (au fur et à mesure que l'on s'éloigne de la station considérée, la qualité de l'ajustement se dégrade jusqu'à devenir inférieure à l'ajustement global). L'exploitation des données des réseaux de pluviomètres d'étalonnage a ainsi donné lieu au développement de procédures d'interpolation relativement sophistiquées (Brandes, 1975, parmi d'autres). Les ajustements locaux sont faits en interrogeant les sites d'étalonnage les plus proches et en leur assignant des poids inversement proportionnels à la distance. On peut aussi modeler les observations du radar avec les données du réseau par une technique d'ajustement plan tenant compte de la variance des précipitations indiquées par le radar. Suivant la densité 


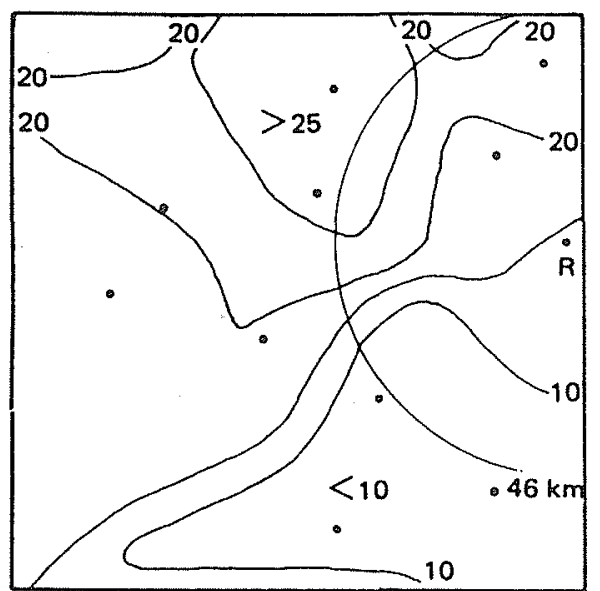

(a)

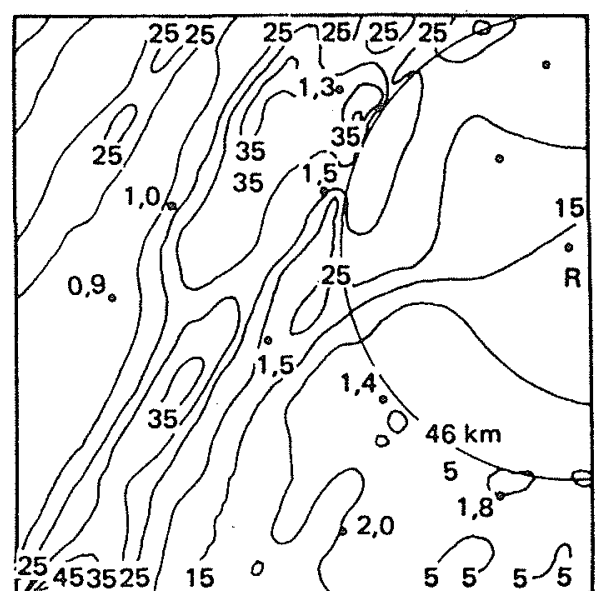

(b)

Figure 7 - a) champ de précipitation au sol déduit des mesures d'un réseau de pluviomètres au sol ; b) le même champ de précipitation déduit de l'ajustement des estimations d'un radar avec les données du réseau de pluviomètres au sol.

des points de mesure au sol, la correction peut inclure les écarts systématiques associés avec l'erreur d'étalonnage du radar, les influences d'échelle synoptique sur la relation $Z-R$, les variations spatiales de petite échelle de $Z-R$ et les modifications des précipitations audessous du faisceau radar. La figure 7 est un exemple du résultat obtenu par ajustement de données radar par un réseau de mesures pluviométriques au sol.

\section{Conclusion}

Globalement, l'erreur dans la mesure de la quantité d'eau atteignant le sol décroît quand la superficie considérée et la durée d'intégration augmentent. Par exemple, pour des superficies inférieures à $50 \mathrm{~km}^{2}$ et des durées d'intégration inférieures à $10 \mathrm{mn}$, l'erreur dans la mesure de précipitation est supérieure à $35 \%$. Le système de mesure constitué par le radar et le réseau de pluviomètres d'étalonnage est supérieur au réseau seul, particulièrement pour les précipitations convectives en averses. Dans les cas courants de pluie de ce type, un radar étalonné par deux points de mesures pluviométriques au sol couvrant un domaine de $1000 \mathrm{~km}^{2}$, autorise la même précision qu'un réseau de 50 pluviomètres répartis sur la même surface soit environ $20 \%$. Sur $1000 \mathrm{~km}^{2}$, la quantité de précipitation intégrée sur 1 heure est estimée à $40 \%$ avec 10 pluviomètres; si l'on y ajoute un radar étalonné, on améliore la précision de $15 \%$.

D'après les résultats concordants des divers auteurs cités, il apparaît que :

- pour des durées d'intégration longues ( $>24$ heures), la mesure des quantités de précipitation, en combinant les données d'un radar et celles d'un réseau pluviométrique, conduit à des erreurs moyennes d'environ 10 à $30 \%$ pour un réseau pluviométrique comportant 1 point de mesure pour 1000 à $2000 \mathrm{~km}^{2}$;

- l'erreur diminue lorsque l'espacement des pluviomètres diminue, et l'intérêt de l'intervention du radar s'amenuise également puisqu'un réseau de pluviomètres seul, s'il est suffisamment dense, conduit à la même précision que les données combinées;

- pour les durées d'intégration supérieures à 24 heures, la limite correspondant à l'égalité de précision entre le réseau seul et la combinaison du réseau et du radar équivaut à un réseau comportant un pluviomètre pour une superficie de 250 à $300 \mathrm{~km}^{2}$;

- pour un réseau de densité supérieure à cette valeur, le radar n'apporte pas d'amélioration dans la précision des estimations mais donne des informations sur la structure fine tridimensionnelle spatiale et temporelle.

Dans tous les cas, le cumul des données sur de longues durées conduit, pour la pluie, à des résultats en accord avec la "relation standard"

$$
Z=200 R^{1,6}
$$

à mieux qu'un facteur 2 près.

La précision des estimations de précipitations de neige à l'aide d'un radar étalonné est semblable à celle que l'on peut atteindre pour les mesures de pluie par la même technique (Collier et Larke, 1978).

La précision des mesures indiquée ci-dessus est celle que l'on sait actuellement obtenir à l'aide d'un radar étalonné par un réseau de pluviomètres. Elle n'est nullement une limite infranchissable et il est vraisemblable que de nouveaux progrès peuvent être réalisés. Parmi les voies d'études nouvelles, les plus prometteuses semblent être la prise en compte de l'état de polarisation des signaux rétrodiffusés qui apporte une information supplémentaire sur la taille des hydrométéores, la mise en œuvre de réseaux de radars assurant une "couverture" plus homogène des champs observés et permettant une prévision à court terme précise (Browning et al., 1982) ou dans un avenir plus lointain l'emploi de radars installés à bord de satellites (Atlas et al., 1978), mais aussi l'utilisation de nouvelles techniques numériques pour le traitement et la présentation des données.

\section{Annexe 1 : Mesures par at ténuation}

Il a été proposé de mesurer l'intensité des précipitations par radar à partir de l'atténuation subie par 
l'écho d'une cible connue observée à travers la précipitation (Juillerat et Godard, 1963; Collis, 1964; Godard, 1965), en utilisant une longueur d'onde courte entre 0,86 et $3,2 \mathrm{~cm}$ ).

On montre qu'entre l'atténuation $a_{p}$ et l'intensité de précipitation $R$, l'on peut écrire une relation de forme :

$$
a_{p}=k R^{\gamma}
$$

où $k$ et $\gamma$ sont des facteurs ne dépendant que de la fréquence et de la température.

La méthode est basée sur la relation quasi-linéaire entre $a_{p}$ et $R$ autour de la longueur d'onde $1 \mathrm{~cm}(\gamma$ voi$\sin$ de 1 ). Cette linéarité implique une relative indépendance de la relation ci-dessus vis-à-vis de la distribution granulométrique de la précipitation. Plus précisément, le terme $\gamma$ pour des longueurs d'onde allant de $0,86 \mathrm{~cm}$ à $3,2 \mathrm{~cm}$ augmente de 1,04 à 1,16 tandis que corrélativement l'erreur moyenne de l'estimation de $R$ à partir de $a_{p}$ augmente de $9 \%$ à $21 \%$, ces accroissements reflétant une moindre indépendance vis-à-vis de la distribution granulométrique avec l'augmentation de la longueur d'onde (Atlas et Ulbrich, 1977). Cependant, même à $3,2 \mathrm{~cm}$ l'erreur demeure faible. La méthode est donc intéressante en raison de sa précision, mais elle ne permet pas la continuité spatiale et n'a pas la souplesse des observations par rétrodiffusion. Elle est applicable pour des mesures à courte distance dans des directions privilégiées (mesures au-dessus d'un site déterminé), mais reste difficilè à mettre en ouvre pour des observations panoramiques sur une grande étendue.

Une seconde méthode faisant appel à l'atténuation par les précipitations mais sans recours à l'écho de cible au sol, utilise un radar à 2 longueurs d'onde, l'une non atténuée, l'autre fortement atténuée (par exemple $10 \mathrm{~cm}$ et $0,86 \mathrm{~cm}$ ou $10 \mathrm{~cm}$ et $3 \mathrm{~cm}$ ). On procède à une mesure directe de l'atténuation entre deux distances le long d'un faisceau commun aux deux longueurs d'onde en considérant le rapport des puissances rétrodiffusées pour chacune d'elles. L'étalonnage des deux radars n'est pas nécessaire (Eccles et Mueller, 1971). Les résultats obtenus sont médiocres.

Un effort supplémentaire est nécessaire pour évaluer plus complètement et optimaliser ces procédés.

\section{REFERENCES}

ATLAS D., and ULBRICH C.W. - Path and area intergrated rainfall measurement by microwave attenuation in the 1-3 $\mathrm{cm}$ band. J. Appl. Meteor., 16, 1977, 1322-1331.

ATLAS D., BANDEEN W.R., SHENK W., GATLIN J.A. and MAXWELL M. - Visions of future operational meteorological satellite system. Proc. of the EASCON 78 Electronics and Aerospace Systems Conf. Arlington, Virginia, 1978.

BRANDES E.A. - Optimizing rainfall estimates with the aid of radar. J. Appl. Meteor. 14, 1975, 1339-1345.

BROWNING K.A., COLLIER C.G., LARKE P.R., MENMUIR P., MONK G.A. and OWENS R.G. - On the forecasting of fontal rain using a weather radar network. Month. Wea. Rev., 110, 1982, 534-552.

CARBONE R.E., and NELSON L.D. - The evolution of raindrop spectra in warm-based convective storms as observed numerically modeled. J. Atmos. Sci., 12, 1978, 2302-2314.

CARTMILL R.H. - Rainfall rate measurement using two radar sets of different wavelengths : Theory. Prep. 10 th Wea. Radar Conf., Amer. Meteor. Soc., Boston, 1963, 265-270.

CATANEO R.A., and STOUT G.E. - Raindrop-size distributions in humid continental climate and associated rainfall raterader reflectivity relationships. J. Appl. Meteor., 7, 1968, 901-907.

COLLIER C.G. - Rainfall measurement by radar. Proceedings sympsium on weather radar and water management. Water Research Centre, Medmenham, England, 1975.

COLLIER C.G., and LARKE P.R. - A case study of the measurement of snowfall by radar : an assessment of accuracy. Quart. J. Roy. Meteor. Soc., 104, 1978, 615-121.

COLLIS R.T.H. - Radar precipitation measurements. Prep. 11 th Wea. Radar Conf., Amer. Meteor. Soc., Boston, 1964 $142-146$.

DONNADIEU G. - Etude des caractéristiques physiques et radioélectriques de la pluie à l'aide d'un spectropluviomètre photoélectrique. J. Rech. Atmos., VIII, 1974, 253-266.

DRUFUCA C. - Radar-derived statistics on the structure of precipitation patterns. J. Appl. Meteor., 16, 1977, 10291035 .
DUMOULIN G., and COGOMBLES A. - A comparison of radar values of precipitation intensities and rainfall rate from raingage. Prep. 12 th Conf. on Radar Meteor., Amer. Meteor. Soc., Boston, 1966, 190-197.

ECCLES P. I, and MUELLER E.A - X-band attenuation and liquid water content estimation by dual-wavelength radar. J. Appl. Meteor., 10, 1971, 1252-1259.

FUJIWARA M. - Raindrop-size distribution from individual storms. J. Atmos. Sci., 22, 1965, 585-591.

Gertzman H.S., and ATLAS D. - Sampling errors in the measurement of rain and hail parameters. J. Geophys. Res. $82,1977,4955-4966$.

GODARD S. - Propriétés de l'atténuation par la pluie des ondes radioélectriques dans la bande $0,86 \mathrm{~cm}$. J. Rech. Atmos., II, 1965, 121-167.

HARROLD T.W. ENGLISH E.J, and NICHOLAS C.A. - The accuracy of radar-derived rainfall measurements in hilly terrain. Quart. J. Roy. Meteor. Soc., 100, 1974, 331-350.

HUFF F.A. - Sampling errors in measurement of mean pricipitation. J. Appl. Meteor., 9, 1970, 35-44.

HUFF F.A. - Evaluation of precipitation records in weather modification experiments. Advances in Geophysics, 15, 1971, 59-134, Academic Press, New-York.

JONES D.M.A. - Rainfall drop-size distribution and radar reflectivity. Res. Rept. $n^{\circ}$ 6, Urbana : Meteor. Lab., 1956, Illinois State Water Survey.

JOSS J. et WALDVOGEL A. - Ein Spektrograph für niederschlagstropfen mit automatischer Auswertung. Pure and Applied Geophysics, 68, 1967, 240-246.

JOSS J., and WALDVOGEL A. - Raindrop size distribution and sampling size errors. J. Atmos. Sci., 26, 1969, 566-569.

JUILLERAT R, and GODARD S. - Etude de la corrélation entre l'atténuation atmosphérique d'une onde E.M. et l'intensité de pluie. Rapport interne, avril 1963, Paris : Lignes Télégraphiques et Téléphoniques.

KLAUS V. - Un spectropluviomètre photoélectrique. La Météorologie, $\mathrm{VI}^{\mathrm{e}}$ série, 5, 1976, 21-32.

MARSHALL J.S., and PALMER W.M.K. - The distribution of raindrops with size. J. Meteor., 5, 1948, 165-166. 
PUHAKKA T. - On the variability of the $Z-R$ relationship related to radar echo pattern. Geophysica, 13, 1974, 103119.

SAUVAGEOT H. - Radarmétéorologie. Eyrolles éditeur, 1982, $296 \mathrm{p}$.

SEKHON R.S., and SRIVASTAVA R.C. - Doppler radar observations of drop-size distribution in a thunderstorm. J. Atmos. Sci., 28, 1971, 983-994.

SELIGA T.A., and BRINGI V.N. - Potential use of radar differential reflectivity measurements at orthogonal polarization for measuring precipitation. J. Appl. Meteor., 15, 1976 , 69-76.

STOUT G.E., and MUELLER E.A. - Survey of relationships between rainfall rate and radar reflectivity in the measurement of precipitation. J. Appl. Meteor., 7, 1968, 465-474.
SULAKVELIDZE G.K., and DADALI Yu. A. - Izmerenie intensivnosti osadkov mnogovolnovym radiolokatorom (La mesure de l'intensité des précipitations avec un radar à plusieurs longueurs d'ondes). Prep. 3 th All-Union Conf. Radar Meteor., Moscow : Hydrometeor. Service, 1968, 31.42.

WEXLER R., and ATLAS D. - Radar reflectivity and attenuation of rain. J. Appl. Meteor., 2, 1963, 276-280.

WILK K.E., and KESSLER E. - Quantitative radar measurements of precipitation. Meteor. Monographs, 11, 1970, 315-329.

WOODLEY W., OLSEN A., HERNDON A., and WIGGER V. Comparison of gage and radar methods of convective rain measurement. J. Appl. Meteor., 14, 1975, 909-928.

WILSON J.W., and BRANDES E.A. - Radar measurement of rainfall - a summary. Bul. Amer. Meteor. Soc., 60, 1979, 1048-1058.

\section{Discussion}

Président: J, LABROUSSE

Le Président remercie M. SAUVAGEOT et invite les assistants qui souhaitent intervenir à prendre la parole.

$M$. OBLED remarque que sur la figure 3 (consacrée à l'évolution de l'écart entre estimation radar et estimation optimale de la pluie en fonction de la distance au radar) les meilleurs résultats sont obtenus aux environs de $50 \mathrm{~km}$ et demande quels sont les effets qui se compensent pour créer cet optimum.

M. SAUVAGEOT précise en réponse que les lobes latéraux du radar introduisent, dans le cas d'obstacles à faible distance, des rayonnements parasites (par exemple des réflexions multiples) qui perturbent la mesure sur les premiers kilomètres, pouvant ainsi expliquer le creux observé sur la courbe. Il note que le radar de Dammartin est un cas représentatif de ce phénomène.
Tout en reconnaissant l'intérêt d'ajuster les données radar sur des pluviographes, $M$. GILET souligne que dans de nombreuses situations, notamment convectives, le radar seul permet d'obtenir de meilleurs résultats que la plupart des réseaux pluvio graphiques qui existent actuellement et sont utilisés, entreautres, pour la prévision des crues.

M. SAUVAGEOT s'estime d'accord avec M. GILET dans la mesure où des indications sur la structure des précipitations autorisent le choix d'une loi $Z=A R^{b}$ adaptée. Les estimations qu'il a fournies concernent l'utilisation d'une loi moyenne : celle de Marshall-Palmer, Il note en outre que les mesures de polarisation des ondes rétrodiffusées autorisent des espoirs importants quant à l'utilisation du radar seul. 